\title{
Pratiques résidentielles des ménages et consommation d'électricité : une approche territoriale de la transition énergétique en région Provence-Alpes-Côte d'Azur
}

\author{
Maximin Chabrol ${ }^{\mathrm{a}}$ \\ Doctorant UMR ESPACE, Université d'Avignon, 84029 Avignon Cedex 01, France
}

\begin{abstract}
Résumé. La consommation d'électricité liée à l'habitat en région Provence-AlpesCôte d'Azur représente un tiers de la facture énergétique régionale. La maîtrise des consommations énergétiques, en particulier des consommations d'électricité, est l'un des piliers évoqués par les pouvoirs publics pour une mise en œuvre de la transition énergétique. Une approche infrarégionale permet de caractériser les comportements des territoires face à cette consommation, d'en rechercher les déterminants et de s'interroger sur les types d'actions à mettre en œuvre. Il est possible de mettre en évidence les contingences locales qui confèrent aux espaces de la région Paca un caractère énergivore du point de vue des consommations d'électricité du secteur résidentiel. La méthodologie développée repose sur une analyse en composantes principales et une classification ascendante hiérarchique pour l'élaboration d'une typologie d'espaces énergivores qualifiés autant par leurs fonctions (résidentielle, activités économiques, tourisme...), que par le type d'habitat qui les caractérise (maisons individuelles, appartements). La cartographie des résultats obtenus permet de territorialiser les types et d'engager une réflexion sur la dimension territoriale de la mise en œuvre de la transition énergétique.
\end{abstract}

\begin{abstract}
Electricity consumption of residential sector in the Provence-Alpes-Côte d'Azur area is a third of the regional energy invoice. The control of energy consumption, especially electricity consumption is a priority in the energy transition policy. A sub-regional approach allows us to characterize the behavior of territories facing the consumption, to investigate the determinants and to examine kind of actions to produce. It is possible to highlight the local specificities that give to the PACA area high energy consumption in residential sector. The methodology based on principal component analysis and hierarchical ascendant classification develops a consumption typology qualified by the specifics functions of spaces (residential, economic activities, tourism...) and by the type of housing (houses, apartments). Mapping results can territorialize types and introduce a reflection about the territorial dimension of the energy transition.
\end{abstract}

La question de la transition énergétique se pose aujourd'hui à des échelles régionale et locale alors que le problème de l'approvisionnement et du transport de l'énergie est resté jusqu'à il y a peu de la responsabilité nationale dans la plupart des pays [9]. Les acteurs locaux sont en attente d'un apport de

\footnotetext{
ae-mail : maximin. chabrol@alumni .univ-avignon.fr
}

This is an Open Access article distributed under the terms of the Creative Commons Attribution License 4.0, which permits unrestricted use, distribution, and reproduction in any medium, provided the original work is properly cited. 
connaissance et d'expertise sur les enjeux de la transition énergétique et de sa déclinaison territoriale. La prise en compte d'un développement durable des territoires implique de reconsidérer également les rapports production/consommation d'énergie et de responsabiliser l'ensemble des acteurs locaux pour les décisions d'investissement, d'exploitation, et aussi de recherche de solutions innovantes. Le territoire est en ce sens une instance déterminante de réflexion, sans que l'on puisse répondre d'emblée à la question de sa granularité (départements, bassins de vie, communautés de communes, autres échelles). La question n'est pas seulement technique et touche à la capacité de mobilisation et de responsabilisation des acteurs locaux de se saisir de la question des enjeux énergétiques et de tenter de la résoudre [3].

La réalisation des schémas régionaux climat-air-énergie (SRCAE) et des plans climat-énergieterritoire (PCET) engage déjà les territoires dans la rédaction de documents de planification visant la mise en ouvre de la transition énergétique. Pour ce faire, les territoires doivent entreprendre un diagnostic de leur équilibre énergétique, des enjeux et des potentiels. Mais une fois les orientations stratégiques établies, comment les mettre en œuvre dans ces espaces d'intervention que sont les collectivités territoriales ? Comment définir des espaces d'actions prioritaires en fonction des différentes thématiques de la transition énergétique?

La maîtrise des consommations énergétiques, en particulier des consommations d'électricité, est l'un des piliers évoqués par les pouvoirs publics pour une mise en œuvre de la transition énergétique. Les consommations d'électricité offrent une clé de lecture des territoires. Aussi dans un contexte où la volonté de mener les territoires vers une transition énergétique s'affirme, il convient de prendre en compte toute la mesure de la dimension spatiale de ce phénomène. Une approche infrarégionale peut permettre de caractériser les comportements des territoires face à cette consommation, d'en rechercher les déterminants et de s'interroger sur les types d'actions à mettre en œuvre. L'analyse proposée ci-après vise à mettre en évidence les contingences locales qui confèrent aux espaces de la région Paca un caractère énergivore du point de vue des consommations d'électricité du secteur résidentiel.

\section{Pratiques résidentielles et consommation énergétique : recherche des espaces énergivores en Paca}

Comment repérer des sous-espaces régionaux caractérisés par des profils particuliers de consommation énergétique ? La méthodologie développée repose sur une analyse en composantes principales (ACP) effectuées à partir de variables portant sur la consommation d'énergie et d'électricité du secteur résidentiel, sur les types de logements, leur mode de chauffage, leur modalité d'occupation et la distance aux centres urbains les plus proches ${ }^{\mathrm{b}}$. Les données liées à l'habitat et au logement proviennent de la base INSEE et celles de consommation, de la base Energ'air.

L'objectif de cette analyse est d'identifier les espaces pour lesquels on observe des corrélations et une covariation entre les variables de consommation et les variables résidentielles.

\footnotetext{
b Consommation d'énergie et d'électricité du secteur résidentiel par logement et par commune en TEP en 2010, consommation d'énergie et d'électricité du secteur résidentiel par ménage et par commune en TEP en 2010, part de résidences principales de type maison par commune en 2010, part de résidences principales de type appartement par commune en 2010, part de résidences secondaires par commune en 2010, part de résidences principales avec chauffage individuel électrique par commune en 2010, part de résidences principales avec chauffage central individuel par commune en 2010, part de résidences principales avec chauffage central collectif par commune en 2010, part de résidences principales occupées par des propriétaires par commune en 2010, part de résidences principales occupées par des locataires par commune en 2010, part de logements vacants par commune en 2010, distance réseau en kms de la commune au pôle de la zone d'emploi à laquelle elle appartient.
} 


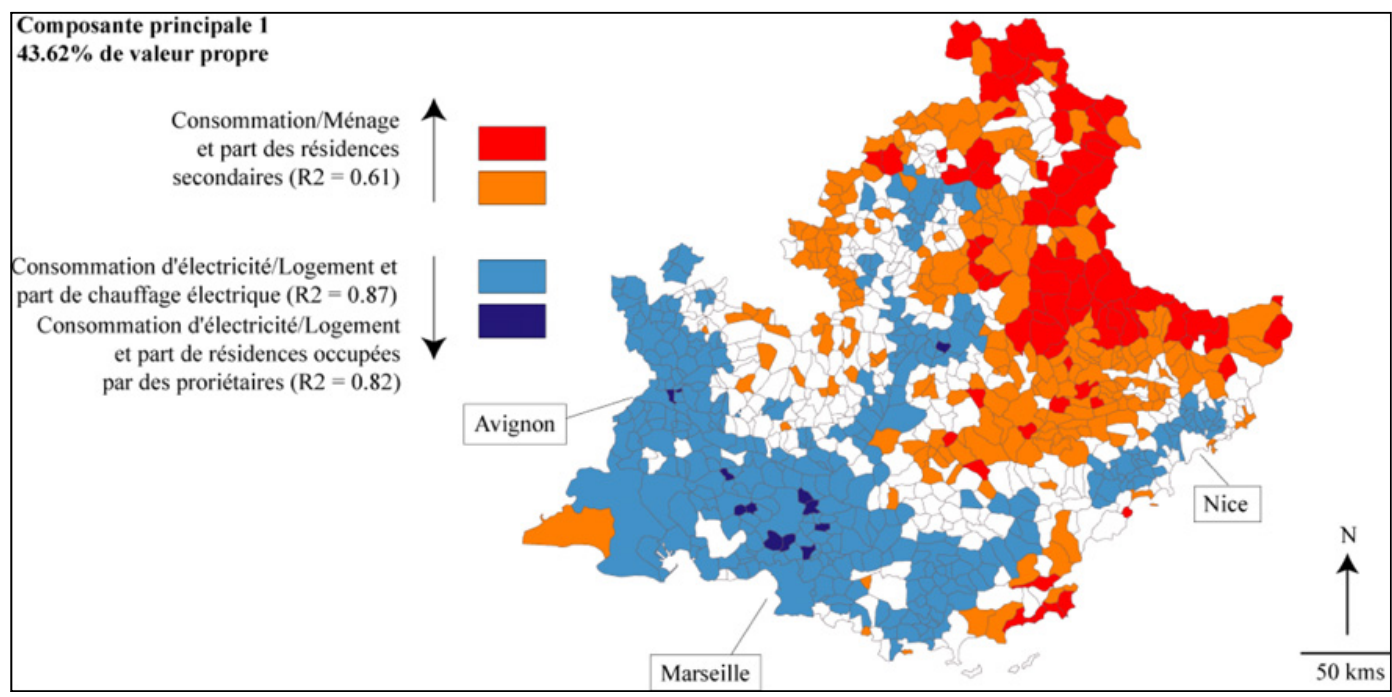

Figure 1. Espaces de la dynamique urbaine et espaces du tourisme.

\section{La dichotomie provençale : espaces de la dynamique urbaine et espaces du tourisme}

Une première analyse en composantes principales sur l'ensemble des neuf cent soixante-trois communes de la région Paca fait apparaître très distinctement deux sous-ensembles qui se différencient par deux fonctions particulières à la région Paca.

Le premier ensemble de communes se situe dans l'arrière-pays et comprend en majorité des communes alpines dont la plupart abritent des stations de sports d'hiver (Fig. 1). À celles-ci s'ajoutent les hauts lieux de villégiature touristique de la Côte d'Azur, du Lavandou à Saint-Tropez, ainsi que la station balnéaire des Saintes-Maries-de-la-Mer. L'analyse en composantes principales décrit dans ces espaces une corrélation entre la part de résidences secondaires et la consommation d'électricité par ménage. Cette observation s'explique par une surreprésentation du nombre de résidences secondaires par rapport au nombre de résidences principales. Les augmentations saisonnières des besoins énergétiques entrainent une surévaluation de la consommation par ménage qui n'est pas représentative de la consommation des ménages résidant à l'année dans ces communes. Cependant, cette particularité confère à ces espaces un caractère énergivore, bien que ponctuel, et soulève des questions en termes de sécurisation de l'approvisionnement énergétique en certaines périodes de l'année.

Le deuxième ensemble de communes correspond aux espaces du dynamisme urbain et économique, à l'exception de la Côte d'Azur et de la bordure littorale des Alpes-Maritimes (Fig. 2). Les résultats montrent des corrélations entre la consommation d'électricité par logement, la part de résidences principales équipées en chauffage individuel électrique et en chauffage central individuel, et la part de résidences principales occupées par des propriétaires.

On peut dès lors supposer que, dans le premier ensemble, la consommation énergétique semble liée à une fonction touristique et dans le deuxième aux fonctions urbaines. Cependant, ce constat paraît biaisé car il correspond à deux spécificités connues du territoire de la région Paca et l'analyse ne peut en rester à cette observation triviale.

Une seconde composante principale ne fait pas apparaître de variables de consommation mais permet de distinguer les espaces caractérisés par une part importante de résidences principales de type maison opposés à un espace caractérisé par une part élevée de résidences principales de type appartement, occupées par des locataires et équipées en chauffage central collectif (Fig. 2). 


\section{SHS Web of Conferences}

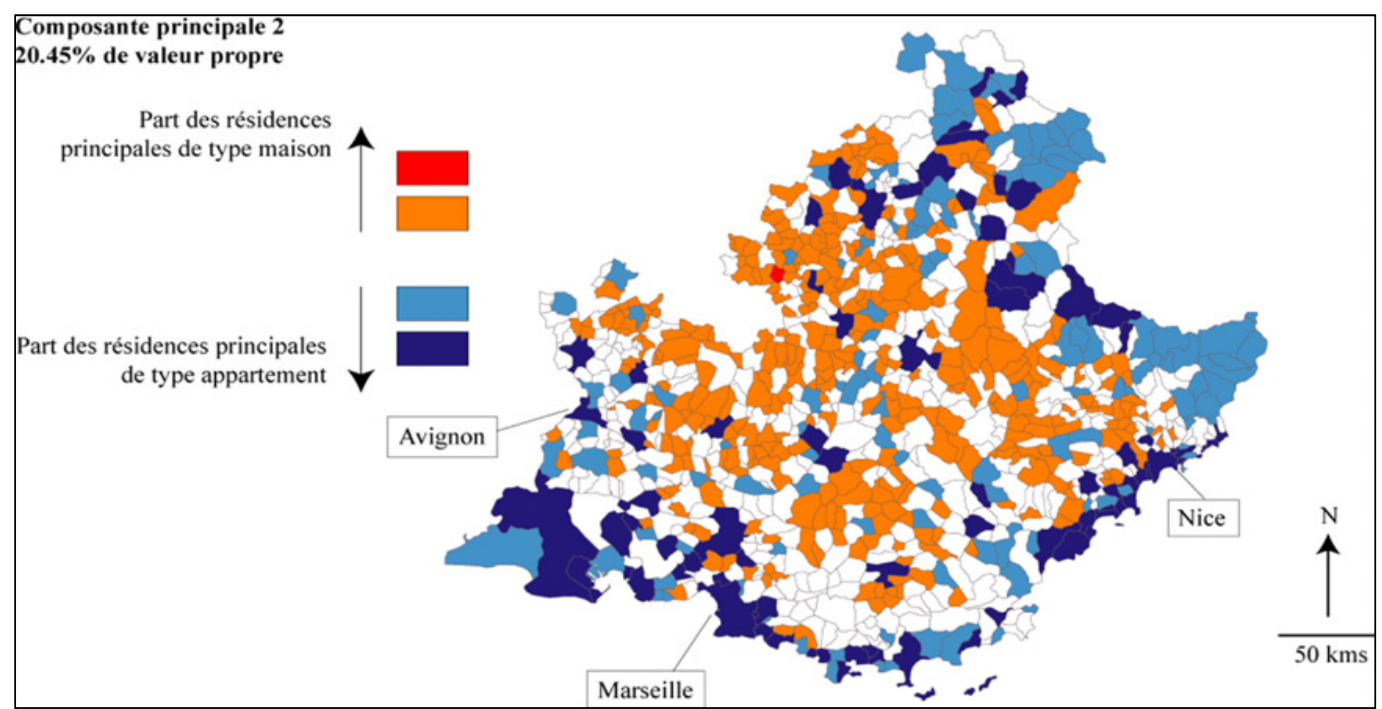

Figure 2. Habitat individuel et habitat collectif.

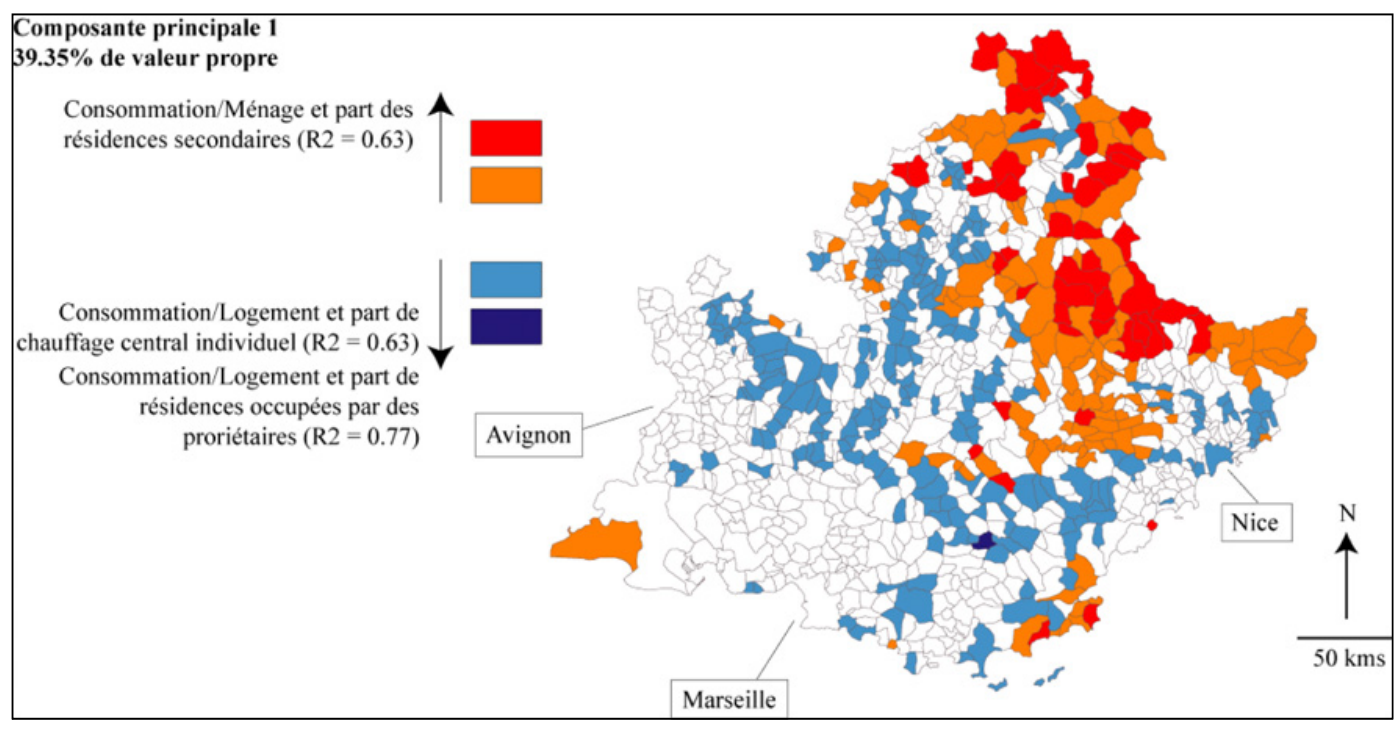

Figure 3. Résidentiel secondaire et habitat individuel.

Une nouvelle analyse est effectuée pour chacun des sous-ensembles définis par la première analyse en composantes principales.

Les espaces du tourisme pour lesquels la part de résidences secondaires et la consommation d'électricité par ménage semblent liées montrent des similitudes avec l'analyse effectuée sur l'ensemble de la région (Fig. 3). On note cependant un degré de précision supplémentaire dans l'identification des communes touristiques. La contribution des stations de sports d'hiver et des communes du Lavandou et de Saint-Tropez, dans la définition de la composante principale sont plus élevées. 


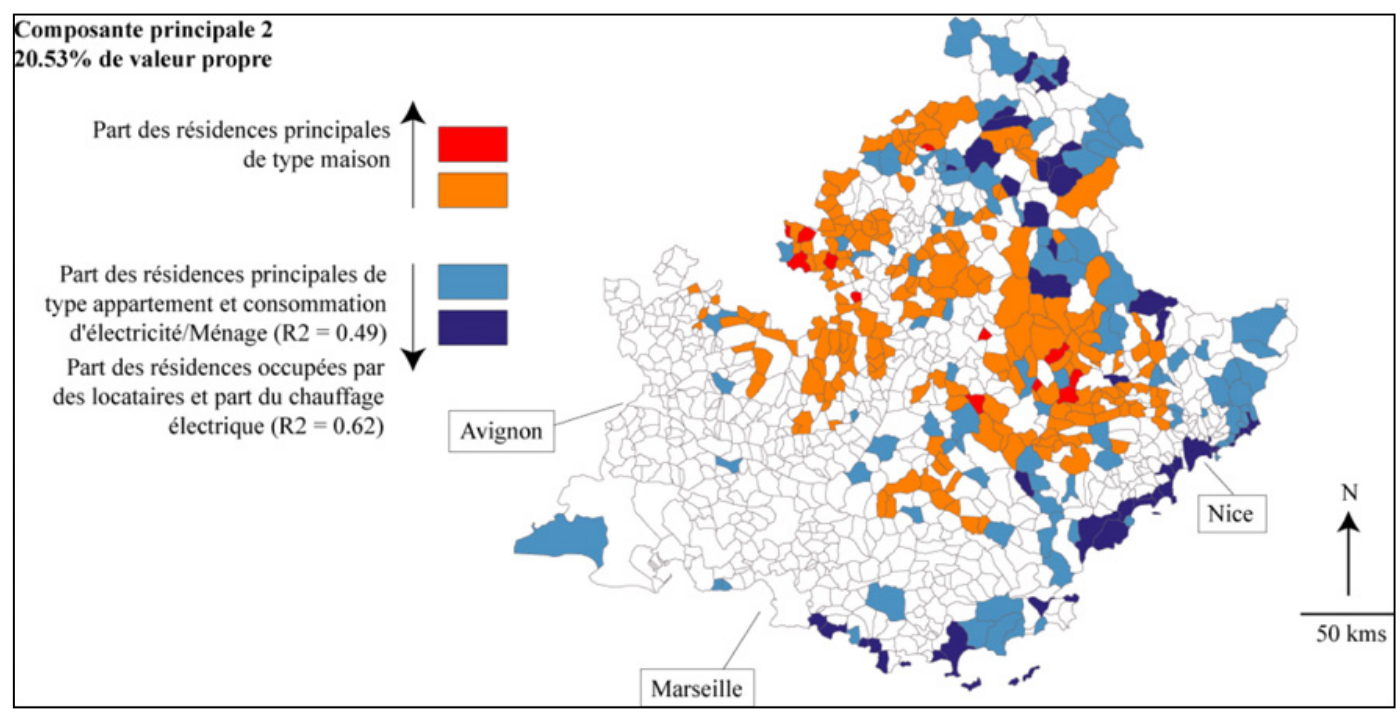

Figure 4. Résidentiel principal individuel et habitat collectif.

La seconde composante principale fait intervenir une variable d'habitat, la part de résidences principales de type appartement (Fig. 4). On observe des corrélations entre cette variable et la consommation d'électricité par ménage, la part de résidences équipées en chauffage individuel électrique, la part de résidences occupées par des locataires et la part de résidences équipées en chauffage central collectif. La part de résidences secondaires n'est en réalité pas liée à la consommation. La consommation est bien liée à des fonctions touristiques mais les résidences secondaires ne sont pas seules représentatives de cette fonction. En effet, on retrouve une nouvelle fois les stations de sports d'hiver et la quasi-totalité de la bordure littorale de la Côte-d'Azur. Toutefois les coefficients de corrélation sont nettement plus faibles. Sous cet ensemble caractérisé par une part importante de résidences principales de type appartement se cachent deux situations différentes : celle des communes alpines caractérisées par un lien entre consommation et fonctions touristiques, par la présence d'habitat locatif de type appartement, et celle d'une partie de la Côte-d'Azur, notamment la région de Nice, caractérisée par de l'habitat collectif à dominante locative.

L'espace du dynamisme urbain fait apparaître certains espaces périurbains plus énergivores que les pôles urbains eux-mêmes (Fig. 5). En effet dans les pôles urbains, les variables résidentielles ne sont pas corrélées aux variables de consommation. Les pôles urbains décrivent une corrélation entre la part de résidences principales de type appartement, la part de résidences principales occupées par des locataires et la part de résidences équipées en chauffage central collectif. En revanche, dans certains espaces périurbains, notamment la périphérie d'Aix-en-Provence, des corrélations élevées apparaissent entre les variables de consommation, la part de résidences principales de type maison individuelle et la part de résidences occupées par des propriétaires. Cela laisse penser qu'habitat locatif, de type appartement, et équipé en chauffage collectif, ne confère pas aux pôles urbains de caractère particulièrement énergivore alors que les espaces du desserrement urbain peuvent apparaître comme tels.

\section{Typologie des espaces énergivores en Paca pour la consommation du résidentiel}

Les analyses précédentes montrent qu'il existe des espaces aux comportements énergétiques particuliers qui peuvent être différenciés par des types de pratiques résidentielles. Les modes d'usage de l'habitat 


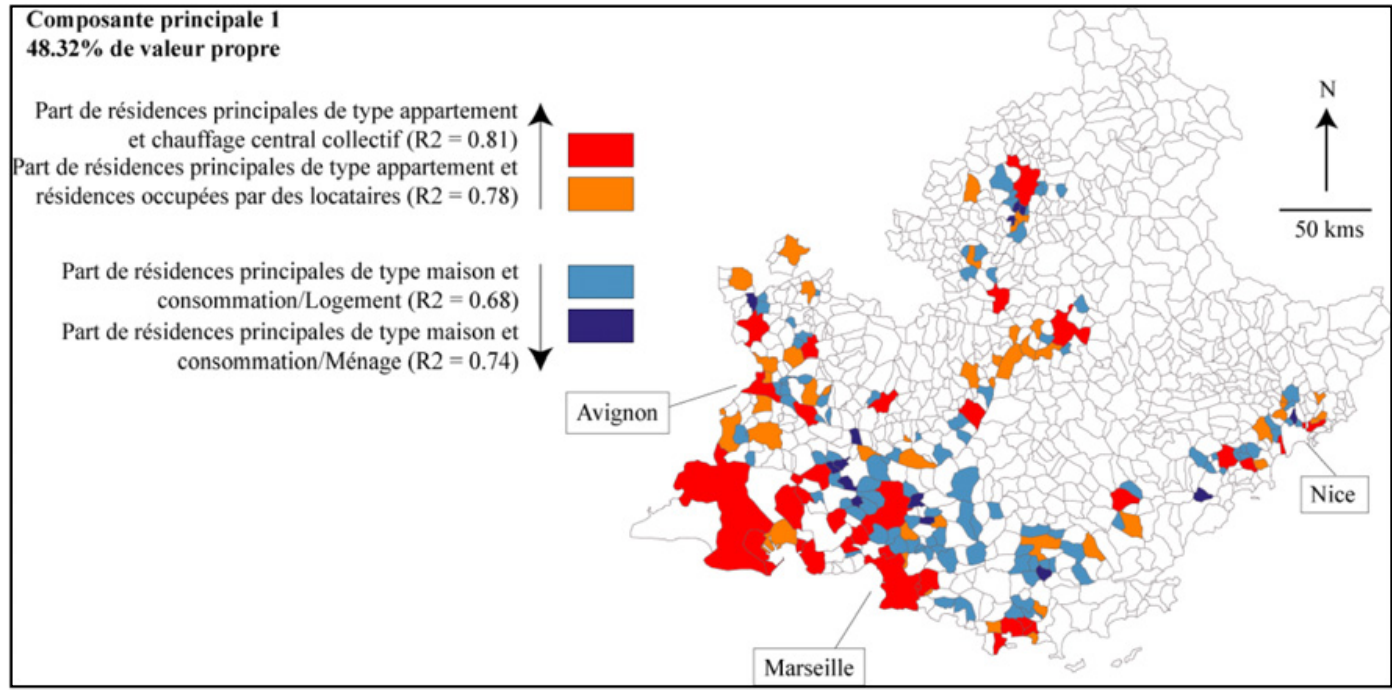

Figure 5. Habitat collectif et habitat individuel.

et son type apparaissent comme des composantes essentielles du caractère énergivore des espaces. Une typologie élaborée à partir d'une classification ascendante hiérarchique $(\mathrm{CAH})$ permet de distinguer ces espaces. La CAH permet de regrouper les neuf cent soixante-trois communes par paquets, en les agrégeant une à une en fonction de leur degré de ressemblance par rapport aux variables spécifiées. Les logiciels de traitement statistique fournissent l'ensemble des partitions possibles lors des étapes d'agrégation. Pour aider à la constitution de la partition de la typologie des espaces énergivores en région Paca et déterminer le nombre de classes le plus pertinent, un certain nombre de paramètres de hiérarchie sont fournis. Parmi eux les indices de niveau des différentes partitions possibles renseignent sur l'inertie intra-classe, donc sur les différenciations des classes entre les partitions successives. Dans notre analyse, un saut important des valeurs de l'indice de niveau, signe d'une différenciation franche entre les classes, est constaté pour la partition en sept classes. Cet indice de niveau de la classification résume près de $70 \%$ de la variance.

Les profils des classes selon les variables (Fig. 6) montrent une opposition entre les communes à la consommation par ménage et à la part de résidences secondaires plus élevées par rapport à la moyenne régionale et celles caractérisées par une consommation par logement et une part du chauffage individuel supérieure au profil moyen des communes de Paca.

La classe 1 est définie par des écarts positifs nets de la consommation par logement et de la part de résidences principales occupées par des propriétaires, et des écarts positifs sensibles de la part de maisons individuelles et de chauffage individuel.

La classe 2 marque une opposition nette entre une surreprésentation de la part d'appartements et une sous-représentation de la part de maisons individuelles. La part de résidences secondaires est bien plus élevée que la moyenne et la consommation par ménage est sensiblement plus élevée aussi.

La classe 3 se caractérise par une part de résidences secondaires, une part de maisons individuelles et une consommation par ménage légèrement supérieure à la moyenne.

La classe 4 se distingue des autres par une consommation nettement inférieure à la moyenne et une part de chauffage collectif et de résidences occupées par des locataires nettement supérieure à la moyenne. 


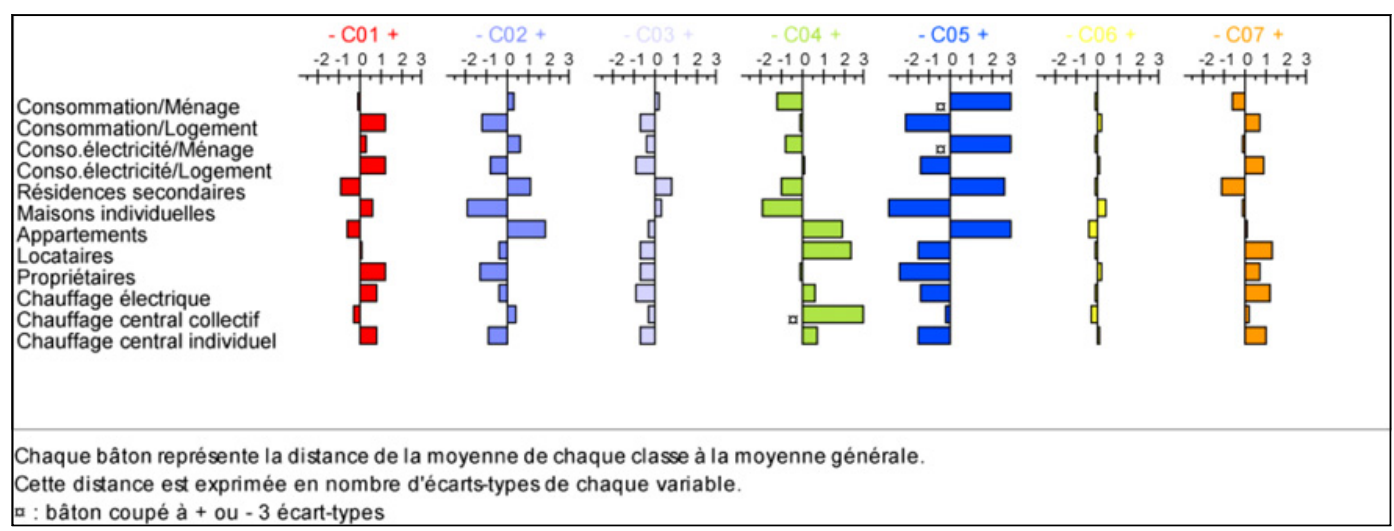

Figure 6. Classes obtenues par classification ascendante hiérarchique.

La classe 5 marque une opposition extrêmement forte entre la part d'appartements et la part de maisons individuelles. La part de résidences secondaires et la consommation par ménage sont beaucoup plus élevées que la moyenne.

La classe 6 fait figure de profil moyen avec une part de maisons et une consommation légèrement au-dessus de la moyenne.

La classe 7 marque une consommation par logement élevée comme la classe 1, mais se distingue de celle-ci par une surreprésentation de la part de locataires et de chauffage individuel électrique.

La cartographie de la typologie présente une synthèse des éléments observés tout au long de l'analyse. Elle permet d'une part de retrouver les éléments majeurs de l'organisation du territoire régional, l'espace du dynamisme urbain et l'arrière-pays, et d'autre part de mettre en valeur les liens entre la consommation énergétique et les pratiques résidentielles particulières à des sous-ensembles régionaux (Fig. 7).

\section{Les espaces périurbains : espace à enjeu, espace de projet}

L'analyse confirme que les pôles urbains ne sont pas des espaces énergivores car l'habitat collectif permet de limiter les consommations (classe 4). Dans le cadre d'une politique de la maîtrise de l'énergie, toute l'attention semble devoir se porter sur les espaces du desserrement urbain qui apparaissent comme un premier espace d'action prioritaire (classes 1 et 7). Le mode de vie caractérisé par la propriété, la maison individuelle de surcroît équipée en chauffage électrique apparaît en région Paca comme un facteur d'une consommation accrue. Cependant le chauffage électrique ne constitue pas un facteur déterminant unique. Les consommations énergétiques ne répondent pas seulement à des besoins matériels comme le chauffage. Elles sont révélatrices des usages qui sont fait des espaces. L'analyse des consommations d'électricité du secteur résidentiel ne peut être réduite au fait qu'un appartement nécessite moins d'énergie pour le chauffage qu'une maison. Aussi un grand nombre de communes caractérisées par une part supérieure à la moyenne de maisons individuelles n'apparaît pas comme particulièrement énergivore (classes 3 et 6). De plus la plupart d'entre elles sont situées en zone d'altitude (classe 6) où les températures hivernales sont faibles. Ce n'est donc pas l'agglomération des individus qui entraîne de fortes demandes en électricité, mais un mode d'habiter l'espace, un mode de répartition des hommes dans l'espace dominé par l'habitat individuel. De nombreuses zones pavillonnaires ont été construites rapidement sans réflexion préalable quant à l'impact possible sur les consommations d'énergie. Cependant les volontés actuelles de réduction de l'étalement urbain sont de plus en plus intégrées aux politiques d'aménagement du territoire, mais ces interventions à posteriori 


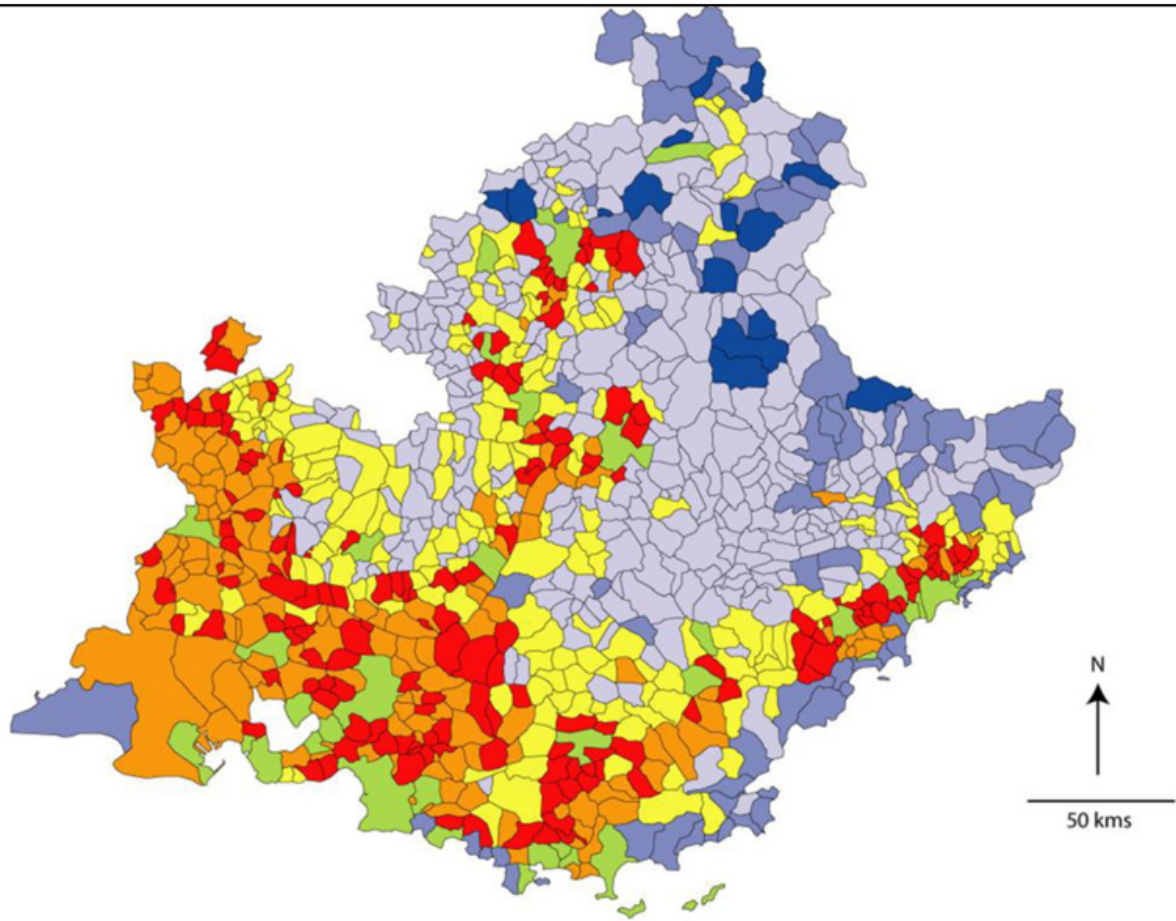

\section{Caractère énergivore des espaces en région Paca}

Consommation très faible, part des appartements, des locataires et du chauffage collectif très élevée.

Consommation et part des maisons légèrement au dessus de la moyenne, part des résidences secondaires relativement élevée.

Consommation par ménage, part des résidences secondaires et part des appartements très élevées.

Consommation par ménage relativement élevée, part des résidences secondaires élevée et part des appartements très élevée.

Profil moyen. part des maisons, de la consommation et du chauffage électrique peu élevée.

Consommation par logement relativement élevée, part des locataires et part du chauffage électrique très élevées.

Consommation par logement et part des propriétaires élevées, part des maisons, du chauffage individuel et électrique relativement élevée.

\section{Profil des classes}

\begin{tabular}{|c|c|c|c|c|c|c|}
\hline 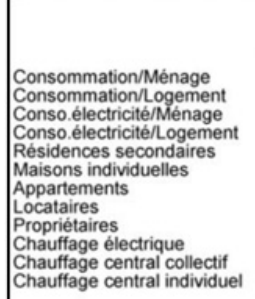 & 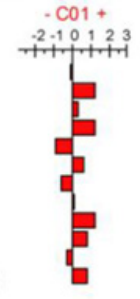 & (1.1.1 & (1.1.1 & (1.1.1.1 & (1.1) & 它 \\
\hline
\end{tabular}

Chaque bâton représente la distance de la moyenne de chaque classe à la moyenne générale.

Cette distance est exprimée en nombre d'écartstypes de chaque variable.

a : bâton coupé à + ou - 3 écart-types

Source : énerg'air, Insee / conception : Maximin Chabrol

Figure 7. Pratiques résidentielles et consommation d'énergie : typologie des espaces énergivores en région Paca. 
sont-elles réellement efficaces face à l'ampleur du phénomène ? On peut s'interroger sur la capacité réelle d'action des pouvoirs publics face à une dynamique territoriale maintenant ancienne et dont le résultat dans l'organisation des territoires est devenu une contrainte. Cela soulève la question du degré de liberté des aménageurs dans l'évolution des systèmes territoriaux. L'aménagement du territoire ne fait-il pas qu'accompagner, au mieux encadrer et contrôler les dynamiques territoriales ? On constate aujourd'hui que les projets liés à la transition énergétique vont dans ce sens. Pour exemple, la commune de Lambesc, située en périphérie d'Aix-en-Provence, typée dans la classe 1 pour laquelle on constate une consommation d'électricité et une part de maisons individuelles élevée, a mis en œuvre une expérimentation d'un réseau électrique intelligent. Ce type de projet devrait aujourd'hui être étudié en amont des réalisations des zones pavillonnaires. Outre les normes récentes sur l'isolation des bâtiments, les projets d'extension urbaine devraient être l'occasion de repenser le développement des territoires dans une perspective de transition énergétique à long terme.

\section{Les espaces du tourisme saisonnier : un modèle de transition?}

Les communes touristiques sont caractérisées par une part d'appartements et une consommation d'énergie très au-dessus de la moyenne. Elles apparaissent comme un deuxième espace d'action prioritaire. Les fonctions des espaces influent sur les consommations énergétiques. Des espaces historiquement attractifs par tropisme solaire comme la Côte d'Azur ou les espaces de villégiature et de tourisme apparaissent aussi comme des espaces à enjeu en termes d'approvisionnement électrique. Ces espaces pourraient devenir de véritables lieux d'expérimentation pour la mise en œuvre de la transition énergétique. Les stations de sports d'hiver sont situées dans des espaces où la ressource forestière est importante. Des projets d'installation de chauffage collectif au bois dans les résidences locatives saisonnières peuvent être envisagés en valorisant la ressource locale et les réseaux de chaleur. De même que l'énergie solaire peut être valorisée dans les stations balnéaires côtières dont la période de forte fréquentation correspond à la période de l'année où le rayonnement solaire est le plus important.

\section{Conclusion et perspectives}

Toute la complexité de la transition énergétique ne réside pas seulement dans la multiplicité des thématiques qu'elle aborde (bâtiment, transport, énergie, ...), mais aussi dans la complexité spatiale qui l'encadre et la détermine, celle de l'organisation des hommes dans l'espace.

Les modes d'usage des espaces et les modes d'habiter l'espace déterminent des comportements globaux de consommations énergétiques. Une analyse régionale permet d'identifier ces comportements et de s'interroger sur les actions à mettre en œuvre à cet échelon territorial. Cependant elle ne permet pas d'identifier dans l'espace régional des comportements individuels guidés par des choix ou contraints par l'appartenance à une catégorie sociale. Une analyse plus fine en termes d'échelle permettrait sans doute de différencier, pour un même type d'espace énergivore, les catégories de population en mesure d'adopter des stratégies résidentielles intégrant la question énergétique, de celles pour lesquelles la notion même de stratégie reste inenvisageable au regard des contraintes fortes que représentent l'accès au logement et à l'emploi.

Cette analyse révèle également le poids de dynamiques territoriales telles que l'étalement urbain, le report de la population vers la périphérie des aires urbaines et le tropisme solaire. On peut s'interroger sur les degrés de liberté dans l'évolution des structures territoriales. Quelles sont les marges de manœuvre réelles des acteurs du territoire et des individus pour intégrer la dimension énergétique dans les stratégies d'aménagement ou dans les stratégies résidentielles individuelles, face aux contraintes exercées par les dynamiques intrinsèques des territoires? 


\section{SHS Web of Conferences}

\section{Références}

[1] M. Béguin, D. Pumain, La représentation des données géographiques (Paris, Armand Colin 1994)

[2] J. Charre, Statistique et territoire (Montpellier, GIP Reclus la documentation française 1995)

[3] J.-M. Chevalier, M. Derdevet, P. Geoffron, L'avenir énergétique : cartes sur table (Gallimard 2012)

[4] M.-H. De Sede, K. Ibrahim, Pour une approche territoriale de l'énergie : une réponse aux défis énergétiques et environnementaux du $\mathrm{XXI}^{e}$ siècle (Festival de géographie de Saint Dié 2007)

[5] A. Grandjean, Vers une société sobre et désirable (Presse Universitaire de France 2012)

[6] Groupe Dupont, L'explication en géographie (Géopoint 2000, 2000)

[7] http://ore.regionpaca.fr/, (s.d.)

[8] http://www.aires-mediterranee.org/html/energair/ (s.d.)

[9] B. Mérenne-Schoumaker, Géographie de l'énergie. Acteurs, lieux et enjeux ( Paris, Belin 2007)

[10] D. Poidevin, La carte moyen d'action (Paris, Ellipses 1999)

[11] Région-Paca, Schéma Régional Air Climat Energie (région PACA 2012)

[12] J. Rifkin, La troisième révolution industrielle (Paris, Les liens qui libèrent 2012) 